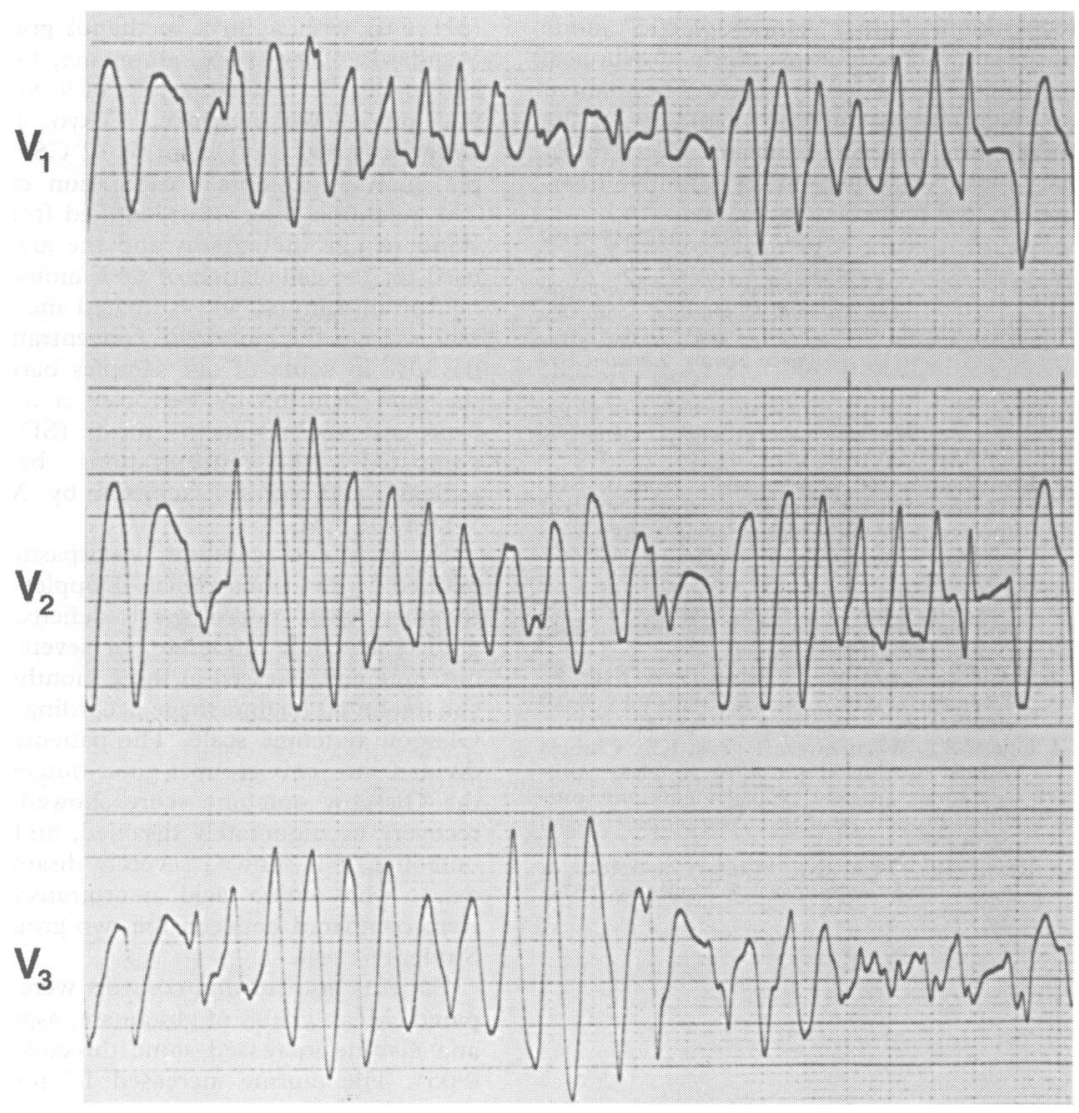

Electrocardiogram showing torsades de pointes. An electromyogram was not obtained because the generalised convulsion ceased.

midbrain circuit, ${ }^{5}$ the present findings are interesting and may assist in the treatment of similar patients.

$$
\begin{array}{r}
\text { TOSHIHIKO KUROIWA } \\
\text { HIROSHI MORITA } \\
\text { HARUSHI TANABE } \\
\text { TOMIO OHTA } \\
\text { Osaka Mishima, } \\
\text { Critical Care Medical Center and } \\
\text { Department of Neurosurgery, } \\
\text { Osaka Medical College, } \\
\text { Osaka, fapan }
\end{array}
$$

Correspondence to: $\mathrm{Dr}$ Toshihiko Kuroiwa, Osaka Mishima Critical Care Medical Center, 11-1 Minami Akutagawa Cho, Takatsuki City, 11-1 Minami A

Osaka 569, Japan.
1 Dasheiff RM, Dickinson LVJ. Sudden unex- pected death of epileptic patient due to cardiac arrhythmia after seizure. Arch Neurol 1986;43:194-6.

2 Leestma JE, Kalelkar MB, Teas SS, et al. Sudden unexpected death associated with seizures: analysis of 66 cases. Epilepsia 1984; 25:84-8.

3 Jay GW, Leestma JE. Sudden death in epilepsy: a comprehensive review of the literature and proposed mechanism. Acta Neurol Scand 1981;63(suppl 82):1-66.

4 Terrence CF, Wisotzkey HM, Perper JA Unexpected, unexplained death in epileptic patients. Neurology 1975;25:594-8.

5 Gilchrist JM. Arrhythmogenic seizures. Diagnosis by simultaneous EEG/ECG recording. Neurology 1985;35:1503-6.

6 Dessertenne F. La tachycardie ventriculaire a deux foyers opposes variables. Arch Mal Cour 1966;59:263-72.

\section{Atypical presentation of vascular events in pituitary tumours: "non-apoplectic" pituitary apoplexy}

Sudden disabling headache, visual deterioration, or impaired conscious level (sometimes in the context of a known pituitary adenoma under medical treatment) is the classical presentation accompanying acute haemorrhage or infarction of the pituitary gland. ${ }^{1}$ We have recently managed three cases in which radiologically and pathologically confirmed vascular events in pituitary tumours presented with ophthalmoplegia in the absence of changes in either visual fields or acuity and in which headache was a minor symptom. This atypical mode of presentation may confound accurate diagnosis and delay appropriate treatment. Surgery for this condition is associated with good examination was normal. Brain MRI showed an intrasellar mass compressing the left cavernous sinus and displacing the optic chiasm (figure). Magnetic resonance angiography performed at the same time excluded an aneurysm. At transphenoidal hypophysectomy infarcted pituitary tissue that was under some tension was evacuated there was a rim of apparently normal tissue evident at the periphery of the lesion. After the operation there was a rapid improvement in the diplopia, which has subsequently completely resolved. Histopathological examination showed infarcted pituitary tissue; there was insufficient viable tumour to allow for immunohistochemistry.

Case 2, a 52 year old man, developed a sudden drooping of his left eyelid accompanied by a mild headache. There had been no alteration in his vision and there was no history of endocrine disorder. Examination showed ptosis and proptosis of the right eye with complete ophthalmoplegia and pupillary involvement. Fundoscopy was normal. Corrected visual acuity was $6 / 9$ on the right and $6 / 5$ on the left; the visual fields were full. Magnetic resonance imaging showed a sellar mass of mixed high signal with suprasellar extension to the optic chiasm, which was clearly compressing the right cavernous sinus and displacing the right internal carotid artery. The appearances were typical of tumour infarction with haemorrhage. At transphenoidal hypophysectomy a large quantity of overtly necrotic pituitary tumour was removed. Histology confirmed necrosis and haemorrhage. Immediately after operation the ocular movements began to improve. At the time of discharge the fourth and sixth nerve palsies had fully recovered but the third nerve palsy remained evident.

Case 3, a 78 year old woman, originally presented with ophthalmoplegia due to a pituitary adenoma. This had almost completely resolved 18 months after transphenoidal hypophysectomy when she developed diplopia of a few days duration accompanied by drooping of the left eyelid. The patient complained of a mild discomfort behind the left eye but no headache. She was alert and lucid. A pupil sparing left third nerve palsy and also a partial left sixth

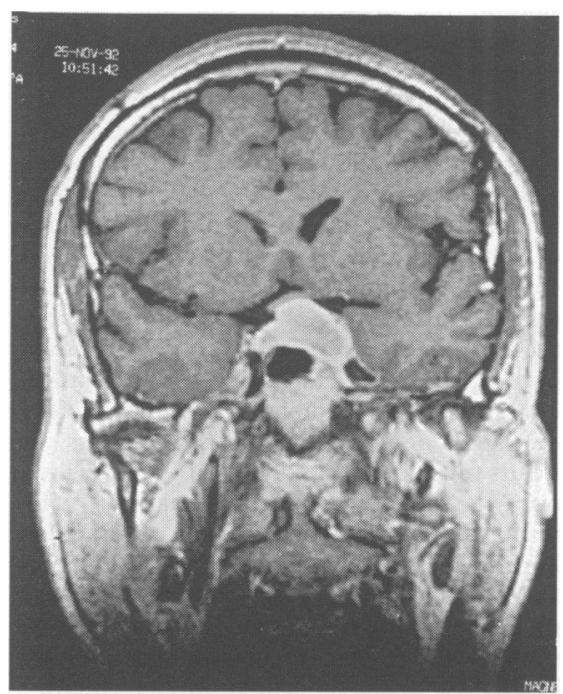

Postcontrast coronal MRI (case 1) showing a homogeneous sellar mass with ring enhancement. There is pronounced compression of the left cavernous sinus. 
nerve palsy were present. Perimetry showed full visual fields and visual acuity was $6 / 9$ in both eyes. Magnetic resonance imaging showed appreciable tumour recurrence. Foci of haemorrhage were evident throughout the lesion, which was compressing both cavernous sinuses, although there was no chiasmal compression. A further endonasal transphenoidal hypophysectomy was performed. Overtly haemorrhagic and necrotic pituitary adenoma was identified at operation and later verified histologically. Two months after this procedure the left third nerve palsy had begun to resolve.

Haemorrhage within pituitary adenomas is a common finding either at the time of operation or on subsequent histopathological examination and is often subclinical..$^{23}$ It has been suggested that in cases of subclinical haemorrhage, tissue necrosis is an uncommon finding whereas it is common in those presenting with clinical apoplexy. Repeated minor haemorrhage within vascular adenomas probably accounts for the clinically occult group, whereas swelling secondary to ischaemic oedema or haemorrhagic infarction in an adenoma which has outgrown its blood supply, is proposed as the more likely sequence of events in cases of clinical apoplexy. ${ }^{4}$ The second mechanism seems to have been responsible for the three cases presented here, where extensive necrosis was uniformly seen. The absence of severe headache, obtundation, and altered visual acuity accords with the finding that whereas ophthalmoplegia is compatible with an alert state, defects in visual acuity are more often associated with impaired consciousness. ${ }^{5}$

The mortality and morbidity of untreated classical pituitary apoplexy is high, whereas the results of expedient surgical intervention for pituitary apoplexy are generally good. Misdiagnosis of pituitary apoplexy even in its more typical presentation is common, however, and it is therefore important that those involved in the initial evaluation of patients are aware of the variability in clinical presentation and the need for early and appropriate radiological evaluation. Magnetic resonance imaging combines high diagnostic accuracy with excellent anatomical detail particularly in the coronal plane (figure).

The case for urgent surgical decompression in the presence of obtundation and actual or threatened visual loss is generally supported. In the alert patient presenting with ophthalmoplegia in the absence of visual field deficit or impaired acuity the surgical role is less clearly defined and many advocate conservative management in this instance. Steroid treatment and close clinical observation is certainly associated with spontaneous improvement in some cases, although there do not seem to be any reliable predictive criteria on which such patients can be identified. The clinical course is unpredictable and the possibility of rapid deterioration with blindness or death must be borne in mind and weighed against the surgical alternative. The transphenoidal approach in experienced hands is extremely well tolerated and surgery ensures immediate decompression of the parasellar structures and affords the opportunity to obtain tissue for histological evaluation to aid subsequent management.

Extraocular muscle palsies may occur as the sole manifestation of infarction or haemorrhage in pituitary adenomas. Early recognition of this "non-apoplectic" mode of presentation allows prompt neurosurgical management, hopefully averting permanent ophthalmoplegia and the possibility of life threatening upward extension of the pituitary mass, while permitting definitive treatment of the underlying adenoma.

DOMINIC THOMPSON MICHAEL POWEL Department of Surgical Neurology,
The National Hospital for Neurology and Neurosurgery, Queen Square, London, UK OLIVER FOSTER Department of Neurology,
The Middlesex Hospital, London, UK

1 Brougham M, Heusner AP, Adams RD. Acute degenerative changes in adenomas of the pituitary body with special reference to pituitary apoplexy. $f$ Neurosurg 1955;7: 421-39.

2 Mohr G, Hardy J. Haemorrhage, necrosis, and apoplexy in pituitary adenomas. Surg Neurol 1982;18:181-9.

3 Wakai S, Fukishima T, Teramoto A, Sano K. Pituitary apoplexy: Its incidence and clinical Pignificance. $\mathscr{F}$ Neurosurg 1981;55:187-93.

4 Onesti ST, Wiseniewski T, Post KD. Clinical versus subclinical pituitary apoplexy: presentation, surgical management, and outsentation, surgical management, and out26:980-6.

5 Rovitt RL, Fein JM. Pituitary apoplexy: a review and reappraisal. $\mathcal{f}$ Neurosurg 1972 ; 37:280-8.

Excitotoxic amino acid neurotransmitters are increased in human cerebrospinal fluid after subarachnoid haemorrhage

Experimental evidence ${ }^{1}$ suggests a role for glutamate neurotoxicity in many neurodegenerative disorders, but few clinical studies have been conducted to substantiate this hypothesis. ${ }^{23}$ We have measured excitatory and inhibitory amino acid neurotransmitters including glutamate, aspartate, taurine, and alanine in human CSF in the acute stage of subarachnoid haemorrhage in relation to clinical outcome.

The study protocol was approved in advance by the local ethics committee and informed consent was obtained from a relative. Ten patients who had a subarachnoid haemorrhage due to a ruptured cerebral aneurysm were included in this study (table) and their ruptured aneurysm was clipped within 48 hours of ictus. A ventricular catheter was placed, CSF was intermittently drained to maintain the intracranial pressure below $20 \mathrm{~mm} \mathrm{Hg}$, and CSF samples were collected from day 3 to day 8 after the haemorrhage and for at least two days after operation. Samples were deproteinised and stored at $-80^{\circ} \mathrm{C}$ until analysis. Control CSF samples were collected and stored in the same way from patients without neurological disorders who underwent spinal taps for spinal anaesthesia.

The CSF samples were diluted 10 times with distilled water and $30 \mu \mathrm{l}$ aliquots were used. Amino acids were measured three times for each CSF sample by high performance liquid chromatography with an electrochemical detector (ECD-100; EICOM, Kyoto, Japan) after a $4.6 \mathrm{~mm}$ (diameter) $\times$ $150 \mathrm{~mm}$ reverse phase precolumn (Eicompak, MA-50DS, EICOM, Kyoto, Japan) derivatisation procedure with $o^{-}$ phthaldialdehyde and ethylmercaptan. The liquid phase was $0.1 \mathrm{~mol}$ phosphate buffer
(pH 6.0) with a $30 \%$ methanol gradient. Standards of aspartate, glutamate, taurine, and alanine in concentrations of 9,90 , and 900 pmol (Wakojunyaku, Tokyo, Japan) were measured every eight to 12 CSF sample analyses to obtain calibration curves. The retention time was identified from the standard chromatograms and the area was used for the calculation of CSF amino acid neurotransmitters. We estimated the maximal error of the glutamate concentration to be $30 \%$ in some of our samples based on the data reported by Ferrarese et al. ${ }^{4} \mathrm{We}$ expressed the results as mean (SD) and compared the concentrations between patients and control subjects by MannWhitney $U$ test.

Symptomatic cerebral vasospasm was defined with transcranial Doppler and, together with neurological deficits, was graded as mild, moderate, or severe. The outcome was assessed at three months after subarachnoid haemorrhage according to the Glasgow outcome scale. The patients were divided into two groups; good outcome, if the Glasgow outcome score showed good recovery or moderately disabled, and poor outcome, if it showed severely disabled or dead. The amino acid neurotransmitters were compared between the two groups by Student's $t$ test.

Patients and normal controls were compared. Mean values of glutamate, aspartate, and alanine increased some threefold $(\mathrm{p}<$ $0 \cdot 05)$. The taurine increased $1 \cdot 7$-fold but the difference was not significant. Five patients made a good recovery, one was moderately disabled, two severely disabled, and two died of delayed ischaemia due to vasospasm (table). Differences in amino acid neurotransmitter concentrations were compared between those who had good outcomes and those with poor outcomes. Concentrations of the amino acid neurotransmitters tended to be higher in the poor outcome group, but these differences were not significant.

Persson et al evaluated the cortical extracellular glutamate concentration in a patient with severe subarachnoid haemorrhage by a microdialysis method ${ }^{5}$ and found a 25 -fold increase in glutamate, aspartate, and taurine under conditions of energy perturbation as indicated by the simultaneously measured lactate:pyruvate ratio and suggested that a massive accumulation of glutamate and aspartate in the extracellular space may contribute to neuronal injury in subarachnoid haemorrhage. ${ }^{5}$ We measured concentrations of amino acids in the ventricular CSF, which can be regarded as a pool into which substances diffuse from the extracellular space. As the amino acids were diluted in the CSF in the ventricles, their concentrations in the extracellular space may be higher than those measured in the CSF

The concentration of extracellular glutamate that is toxic to neurons is poorly defined particularly as any such effects are enhanced by compromising energy generation. ${ }^{6}$ In vitro experiments with cultured neurons have suggested that concentrations of 5-100 $\mu \mathrm{mol} / 1$ glutamate are toxic to neurons. ${ }^{1}$ Olney et al reported that direct injection of $500 \mathrm{nmol}$ (as $1 \mu \mathrm{l}$ of a $500 \mathrm{mmol} / 1$ glutamate solution over five minutes) into the rat striatum was necessary to cause delayed local neuronal degeneration. ${ }^{3}$ The relation between the amount of increase of amino acid neurotransmitters in CSF and 